Aquaculture

Elsevier Editorial system(tm) for

Manuscript Draft

Manuscript Number: AQUA-D-14-00373R2

Title: Relationship between uninflated swim bladder and skeletal anomalies in reared gilthead seabream (Sparus aurata)

Article Type: SI: larvi 2013

Keywords: skeletal anomalies, skeletal deformities, swim bladder anomaly, sparus aurata, meristic counts

Corresponding Author: Dr. Clara Boglione, AM in Biological Sciences

Corresponding Author's Institution: University of Rome Tor Vergata

First Author: Loredana Prestinicola, PhD

Order of Authors: Loredana Prestinicola, PhD; Clara Boglione, AM in Biological Sciences; Stefano Cataudella, Professor

Abstract: The higher mortalities and lower morphological quality of juveniles with a non-inflated swim bladder still remain a major problem in intensive farming. The aim of this study was to investigate a possible relationship between the presence of an abortive swim bladder and skeleton anomalies in gilthead seabream juveniles. A batch of 109 DAH (LS range: 16-47 mm) gilthead seabream was split into two groups on the basis of the presence (group SB)/absence (WSB group) of a normally inflated swim bladder, and processed for skeletal anomaly and meristic count analysis. The presence of pectoral fin anomalies and the absence of any clear-cut and sound relationship between abortive swim bladder and axis deviations characterize the WSB group. Rarer anomalies, such as scoliosis and lordosis in the pre-haemal vertebrae, kyphosis in the haemal vertebrae, pre-haemal and haemal vertebrae body anomalies, exclusively affected the WSB seabream. This result contradicts what is observed in other species, where abortive swim bladder is shown to cause axis deviation.

The authors hypothesize that when gilthead seabream larvae with uninflated swim bladder encounter difficulty in maintaining the level in the water column, they have to overuse flapping of the pectoral fins (which appear more deformed in the seabream without swim bladder of this study). This determines a hyper-activity of the pectoral muscles, which then exerts an intense mechanical load on pre-haemal muscles and ossifying vertebrae. Vertebrae and axis anomalies in the pre-haemal and in haemal (in this study still rare in the WSB lot) regions may be later effects, as postural consequences due to the uninflated swim bladder. 
Cover Letter

Rome, 12/06/2014

Dear Editor,

Please receive the last version of our research article (AQUA-D-14-00373) entitled: "Relationship between uninflated swim bladder and skeletal anomalies in reared gilthead seabream (Sparus aurata)" to be considered for publication in Larvi' Special Issue.

According to the reviewers' comments, the paper has been revised By Prof. Ian Mc Gilvray.

We'll be waiting for any further instruction from your Editorial Office,

Sincerely yours,

Best regards

On behalf of all co-authors,

Dr. Clara Boglione

"Tor Vergata" University of Rome - Biology Department

Via della Ricerca Scientifica s.n.c., 00133 Rome (Italy) 
According to the reviewers' comments, the paper has been revised By Prof. Ian Mc Gilvray.

Response to Reviewers

According to the reviewers' comments, the paper has been revised By Prof. Ian Mc Gilvray. 


\section{HIGHLIGHTS}

- A non-inflated swim bladder still remains a major problem in intensive farming

- Mismatched results of the different authors describe an unclear context

- Fish without swim bladder has higher frequency of anomalies of pectoral fin

- Absence of any clear-cut and sound relationship between abortive swim bladder and axis deviations

- Abortive swim bladder compels larvae to overuse of pectoral fins, thus pectoral muscles could create a extra-load on differentiating pectoral elements at first, and on pre-haemal vertebrae later in the development. 


\title{
Relationship between uninflated swim bladder and skeletal anomalies in reared gilthead seabream (Sparus aurata)
}

Loredana Prestinicola, Clara Boglione*, Stefano Cataudella.

Department of Biology, University of Rome “Tor Vergata”, Via della Ricerca Scientifica, 00133 Rome, Italy.

*Corresponding author email: boglione@uniroma2.it

\begin{abstract}
The higher mortalities and lower morphological quality of juveniles with a non-inflated swim bladder still remain a major problem in intensive farming. The aim of this study was to investigate a possible relationship between the presence of an abortive swim bladder and skeleton anomalies in gilthead seabream juveniles. A batch of 109 DAH (LS range: 16-47 mm) gilthead seabream was split into two groups on the basis of the presence (SB group)/absence (WSB group) of a normally inflated swim bladder, and processed for skeletal anomaly and meristic count analysis. The presence of pectoral fin anomalies and the absence of any clear-cut and sound relationship between abortive swim bladder and axis deviations characterize the WSB group. Rarer anomalies, such as scoliosis and lordosis in the pre-haemal vertebrae, kyphosis in the haemal vertebrae, pre-haemal and haemal vertebrae body anomalies, exclusively affected the WSB seabream. This result contradicts what is observed in other species, where abortive swim bladder is shown to cause axis deviation. The authors hypothesize that when gilthead seabream larvae with uninflated swim bladder encounter difficulty in maintaining the level in the water column, they have to overuse flapping of the pectoral fins (which appear more deformed in the seabream without swim bladder of this study). This determines a hyper-activity of the pectoral muscles, which then exerts an intense mechanical load on pre-haemal muscles and ossifying vertebrae. Vertebrae and axis anomalies in the prehaemal and in haemal (in this study still rare in the WSB lot) regions may be later effects, as postural consequences due to the uninflated swim bladder.
\end{abstract}




\section{Introduction}

The swim bladder is an important organ contributing to the ability of a fish to maintain neutral buoyancy. It develops as an invagination of the digestive tract and its initial gaseous inflation occurs in some fish species, such as striped bass Morone saxatilis, gilthead seabream Sparus aurata (Chatain and Ounais-Guschemann, 1990), European seabass Dicentrarchus labrax (Boglione et al., 1995; Chapman and Hubert, 1988), walleye Sander vitreus (Marty et al., 1995), striped trumpeter Latris lineata (Trotter et al., 2005) and Chinese sturgeon Acipenser sinensis (Watanabe et al., 2008), when the larvae gulp air at the water surface. In many marine finfish species swim bladder inflation occurs during a finite 'window' period of early larval development (Woolley and Qin, 2010). Anomalies in the activation and inflation of the swim bladder have been extensively described since the beginning of European aquaculture (Battaglene \& Talbot, 1990; Bennet et al., 1987; Bulak and Heideinger, 1980; Chatain, 1986; Chatain \& Ounais-Guschemann, 1990; Doroshev and Cornacchia, 1979; Giavenni and Doimi, 1983; Marino and Boglione, 1990; Nash et al., 1977; Paperna, 1978; Weppe and Bonami, 1983) but the aetiology of swim bladder inflation anomalies has not been fully determined, with culture conditions more commonly addressed as contributory factors. Many factors actually influence initial swim bladder inflation, such as air intake and surface access (Trotter et al., 2005; Woolley and Qin, 2010; Yamaoka et al., 2000), photoperiod and light intensity (Battaglene and Talbot, 1990; Fielder et al., 2002; Trotter et al., 2003; Woolley and Qin, 2010), temperature and larval body size (Marty et al., 1995; Trotter et al., 2003; Woolley and Qin, 2010) and salinity (Battaglene and Talbot, 1990; Woolley and Qin, 2010). The most widely accepted cause of the failure of swim bladder inflation is the presence of a superficial oil film in the tank that prevents larvae from gulping air (Chatain, 1989). For this reason, commercial hatcheries routinely use surface skimmers or aeration points located at four points of the surface area to remove the superficial oil film. Both these devices are designed to concentrate the oil film in a small area where it can be manually removed. Nonetheless, some individuals or batches still fail in swim bladder inflation. Peruzzi et al. (2007) illustrated how paternally and maternally inherited factors can contribute to the expression of swim bladder anomalies in sea bass, along with major environmental clues.

Losses at a commercial level, as a result of swim bladder anomalies, are typically in the order of 5$10 \%$ of all fingerlings produced, but can be as high as 50\% in some cases (Woolley and Qin, 2010). In new candidate species for aquaculture, where rearing technologies are poorly developed, the incidence of swim bladder non-inflation can be as high as 70-100\% (Trotter et al., 2001), thus indicating that optimal rearing conditions are required for a successful inflation. 
A non-inflated (or abortive) swim bladder reduces the growth and survival of larvae (Battaglene and

Talbot, 1990; Woolley and Qin, 2010) because it consistently alters the capability of the fish to maintain its position in the water column, causing the fish to descend to the tank bottom and mass mortality from suffocation or infection from the high bacteria loads on the tank bottom, associated with the high density of intensive aquaculture (Woolley and Qin, 2010).

In addition, a high incidence of skeletal anomalies in gilthead seabream, European seabass Dicentrachus labrax, red seabream Pagrus major, yellowtail amberjack Seriola lalandi and Japanese seabass Lateolabrax japonicus lacking a functional swim bladder has been reported by many authors (Boglione et al., 1995; Chatain, 1994; Chatain and Ounais-Guschemann, 1990; Daoulas et al, 1991; Divanach et al., 1997; Kitajima et al., 1981; Paperna, 1978), suggesting a relationship between skeleton and swim bladder anomalies. Curiously, the skeletal anomalies related to the presence of an uninflated swim bladder differed according to the species or to the author. In Japanese seabream Chrysophrys major (now Pagrus major), European seabass and gilthead seabream, the absence of a correctly inflated swim bladder was found to be associated with kyphosis and lordosis (Boglione et al., 2001, 1995; Chatain 1994; Kitajima, 1978; Kitajima et al., 1981, 1977; Iseda et al., 1979;) and calculosis (Boglione et al., 1995; Modica et al., 1993). Conversely, Andrades et al. (1996) found no such correlation in gilthead seabream: they observed that lordotic larvae were already detectable before the inflation of the swim bladder, suggesting that other causes during embryonic development may be implicated in the onset of lordosis. In European seabass, Divanach et al. (1997) observed differences in the position of the centre of lordosis between fish with and without swim bladder: the differences (lordosis in the last pre-haemal vertebra in fish without swim bladder and on the anterior haemal ones in fish with swim bladder) were explained by the authors as a difference in the centre of gravity due to the intense muscular activity of the posterior part of the body in reaction to the sinking tendency in fish without swim bladder. In the same species, Boglione et al. (1995) found significantly higher occurrences of kyphosis in pre-haemal vertebrae, associated with lordosis in the haemal vertebrae and urinary calculi in juveniles with uninflated swim bladder.

The mismatched results of the different authors suggest an unclear context, mainly due to the different methodologies, the species or the size/age of samples used by authors for monitoring the skeletal anomalies.

In this framework, this paper reports for the first time a monitoring of skeletal anomalies and meristic count variations in juveniles of gilthead seabream with and without an inflated swim bladder. The aim was to detect a possible relationship between the absence of swim bladder and some skeletal bone anomalies, using a standardised protocol (Boglione et al., 2009, 2006, 2003, 
2001; Prestinicola et al., 2013) for monitoring skeletal anomalies.

\section{Materials and methods}

A total of 287 reared seabream juveniles from a commercial farm were analyzed (day 109 after hatching, DAH), 50 and 237 of which were specimens respectively with or lacking a correctly inflated swim bladder. Each individual was lethally anaesthetized (ethylene glycol-monophenyl ether, Merck, $0.5 \mathrm{ml} \mathrm{L}^{-1}$ ), fixed in $10 \%$ formalin buffered with phosphate buffer ( $\mathrm{pH} 7.2,0.15 \mathrm{M}$ ) and double-stained in toto for cartilage and bone, according to Dingerkus and Uhler (1977). For each individual, two different and independent operators measured the standard length (mm) and performed the skeletal anomaly and meristic count analyses. Standard length (SL; mm) was measured from the tip of the snout to the distal edge of the hypural bones (Kohno et al., 1983), rounded to the higher $0.5 \mathrm{~mm}$. Observations were performed on both sides under a stereomicroscope (Wild, LEITZ). Other details on the two lots analyzed are given in Table 1.

Skeletal anomalies were classified with a dichotomic indicator, where the letter indicates the skeletal element affected, the number and the typology of the anomaly (Table 2). Some anomalies displayed different degrees of alteration (see, for example, C3 and $\mathrm{C} 3^{*}$ ) and were indicated as distinctive variables. Anomalies that affect external body shape or that reduce performance (survival, ability to escape from predators, etc.) of the individual were considered as severe anomalies. Vertebral body fusions are considered as severe anomalies even when the vertebral axis is not deviated (thus not affecting the external shape of the fish). .

The meristic characters considered in the counts were the total vertebrae (including the urostyle), the fin rays (divided into spines and soft rays, upper and lower principal caudal rays), the elements that support fins (pterygiophores, hypurals, epurals, radials) and predorsal bones. Counts of the pair of fin rays were carried out on the left and right sides.

The analysis was carried out making some assumptions: i) non-completely fused bone elements were counted as distinct elements; ii) supernumerary bones with a normal morphology were not considered as anomalies but only as a meristic count variation; conversely, anomalous supernumerary elements were included in the anomalies count (i.e., typologies $5^{*}, 6^{*}, 23$ ); iii) only the clearly and unquestionably identifiable variations in shape were considered as skeletal anomalies: if any doubts arose, then the shape variation was not considered anomalous; iv) misalignment of vertebrae was considered as lordosis and/or kyphosis only if it involved anomalous or fused vertebral bodies; otherwise, it was considered as vertebrae displacement (never observed in this study).

The anatomical terminology is according to Harder (1975) and Matsuoka (1987), with the exception 
of terminology for caudal fin structures, which is according to Schultze and Arratia (1989).

Data obtained from meristic counts were compared with those referring to 5 conspecific wild lots (426 individuals; see Table 3), belonging to LESA (Laboratory of Experimental Ecology and Aquaculture of the University Tor Vergata) historical database, and used here as quality standard of reference (wild-like phenotype, according to Cataudella et al., 2002).

Data obtained from skeletal anomalies evaluation were used to calculate the following derived variables, for each lot:

1. relative frequency (\%) of anomalous individuals;

2. average anomalies load (total number of anomalies/number of anomalous individuals);

3. relative frequency (\%) of individuals with at least one severe anomaly;

4. frequency $(\%)$ of severe anomalies observed out of the total observed anomalies;

5. severe anomalies load (number of severe anomalies / number of individuals with severe anomalies);

6. relative frequency (\%) of each anomaly typology, relatively to the total anomalies observed in each lot;

7. relative frequencies $(\%)$ of individuals affected by each type of anomaly.

The Mann-Whitney U Test was applied to test the statistical significance of differences in the skeletal anomalies between lots on a quantitative basis, using data on anomalies frequencies in each individual, in each lot (Past version 2.14, Hammer et al., 2001).

Data obtained from analysis of skeletal anomalies were transformed into a binary matrix (hereafter named BM: presence of each skeletal anomaly typology $=1$; absence $=0$ ) and subjected to Correspondence Analysis (CA; Benzecri, 1973), in order to represent the qualitative ordination of the two lots and their correspondence with the ordination of anomalies. The CA was applied excluding the anomaly 'absence of swimbladder' (typology 12), in order to assess mainly the differences in the skeletal anomalies between lots.

\section{Results}

The ranges of variation for each meristic character for each lot are given in Table 4.

The two lots showed no differences compared with the wild counts in some characters (i.e. vertebrae, anal rays and pterygiophores, pectoral left side rays, pectoral left and right radials and left pelvic rays). However, WSB seabream displayed a larger number (8) of characters out of the range observed in wild lots, with respect to SB fish (6). The characters with a high number of individuals out of the reference range were the hypurals and epurals in both lots: SB displayed $64 \%$ and $4 \%$ of 
individuals bearing 6 and 7 hypurals, respectively, while WSB displayed $71.7 \%$ and $0.8 \%$, respectively; $24 \%$ of individuals displayed 5 epurals in SB, while WSB displayed $2.1 \%$, $19 \%$ and $2.1 \%$ of individuals with 2,5 and 6 elements, respectively (data not shown). Few individuals with the number of caudal upper rays ( 2 with 7, and 1 with 10 elements in the WSB lot; wild range: 8-9) and predorsal bones (2 individuals with 4 in SB; 2 individuals with 4 elements in the WSB group, vs 2-3 bones in wild lots) outside the range found in wild specimen were observed in both lots (data not shown). The number of principal caudal rays was more variable in WSB (14-20) than in SB and wild (16-18) seabream, but this was due to very few (4) individuals. The other characters showed a similar trend among lots (data not shown).

The median value found to be different among lots was only the number of vertebrae, namely 25 in the SB lot (56\% of individuals) and 24 in the WSB lot (52.3\% of individuals).

As far as skeletal anomalies are concerned, a total of 51 different anomaly typologies were observed, 14 of which belonging to severe typologies. All the 51 typologies were present in the WSB lot, only 35 in SB seabream. Some anomalies are shown in Figs. 1 and 2.

Both lots displayed $100 \%$ of individuals affected by at least one (severe or light) anomaly, while the average load of anomalies was lower in SB (8.8) than in WSB (10.8). The frequency of individuals with at least one severe anomaly was $100 \%$ in WSB and $42 \%$ in SB. This is a trivial datum as expected owing to the presence in all the WSB seabream of the severe anomaly 12 (absence of swim bladder), but if this anomaly is removed (WSB* in Table 5) from the computation procedure, the value remains higher in WSB than in SB (48.5\% vs 42\%, Table 5).

The ratio of severe anomalies to the total detected anomalies was higher in WSB (both considering and not considering the anomaly 12) than in SB, as well as the severe anomalies load. All regions in both lots were found to be affected by anomalies, with pelvic and anal fins displaying the lowest occurrences. In both lots, the most affected region was the haemal vertebrae region (with an incidence of anomalies of $30.1 \%$ in SB and $29.3 \%$ in WSB), followed by that of the caudal fin ( $26 \%$ in SB and $21.1 \%$ of anomalies in WSB). The distribution of the anomalies in the different body regions was similar in the two lots, with the exception of the pectoral fin region, which showed a higher frequency of anomalies in WSB.

The high frequency (higher than 13\%) of anomalies in the haemal vertebrae was mainly due to light anomalies affecting neural (C5, C5*) and haemal arches (C6, C6*) in both lots (see Table 6); the anomalies in the caudal region were mainly hypurals (G9: 5.2\% in SB and 4.3\% in WSB) and epural fusions (G10: $10.7 \%$ in $\mathrm{SB}$ and $8.6 \%$ in WSB), and the presence of supernumerary bones (G23: 5.2\% and 4.1\%, respectively in SB and WSB seabream). 
A more detailed analysis is supplied for each lot below and in Table 6:

- $\quad$ in SB juveniles, 35 typologies of anomaly were detected. Many anomalies were observed only in one or two individuals (i.e. E8L, E21R, F8, etc.), while others affected larger numbers of individuals (i.e. B5, C5, and C6). Scoliosis (S), lordosis (type 2) and kyphosis (type 1) were not observed, while partial (D3) and total (D3*) fusions, and anomalies of vertebral bodies (D4) were observed in the caudal region of 1 (=2\% of individuals), 4 (=8\%), and 7 $(=14 \%)$, individuals, respectively. In addition, anomalous pre-maxillary/maxillary (anomaly code: 14), dentary (anomaly code: 15) and opercular plates (anomaly codes: 17L and 17R) were observed in 2 (=4\% of individuals), 1 (=2\% of individuals), and $9(17 \mathrm{~L}=10 \%$; L17R= $10 \%$ ) individuals, respectively. The anomaly 17 monolaterally affected the left side in 4 individuals and the right one in another 4; only one individual showed bilaterally deformed opercular plates;

- the WSB juveniles displayed 51 different typologies, including the typologies observed in the SB. Obviously, anomaly 12 was detected in $100 \%$ of the juveniles, representing the discriminating factor between the two lots. Other anomalies affecting a large number of individuals were those affecting the neural (D5 and D5*: $12.2 \%$ of individuals; C5 and C5*: 57\% of individuals) and haemal spines (D6 and D6*: 26.2\%; C6 and C6*: $62.9 \%$ of individuals), hypurals (G9 and 19: 44.7\% of ind.) and epurals (G10: 68\% of ind.). Further, $28.7 \%(\mathrm{E} 11 \mathrm{~L})$ and $22.8 \%(\mathrm{E} 11 \mathrm{R})$ of individuals were affected by anomalies of the pectoral rays (vs 4.6 and $4.7 \%$ in SB lot, respectively). Scoliosis (S), kyphosis (C1) and lordosis (B2) were detected only in this group but they were rare, being observed in 6 (S: $2.5 \%$ of individuals), 2 (C1: 0.8\%) and 2 (B2: 0.8\%) individuals, respectively. Rare anomalous vertebral bodies were observed in all vertebrae regions (B4, C4, D4, affecting $17.7 \%$ of individuals), except in the cephalic one (no A4). Anomalous pre-haemal (B4: 0.8\%) and haemal (region C: $3.8 \%$ ) vertebral bodies were exclusive of this lot. Total (D3*) and partial (D3) fusions were observed only in the caudal vertebrae, respectively in $8 \%$ and $5.1 \%$ of individuals, at the same location and with the same occurrences as observed in the SB group. Only three individuals $(1.3 \%)$ were found to be affected by urinary calculosis (typology 13 , versus none in the SB group). The occurrences of anomalous maxillary, dentary and opercular plates were equal to the ones registered in the SB group.

The Mann-Whitney U test, applied to data on anomalies frequencies in each lot, indicated that lots were significantly different as regards incidence of anomalies ( $\mathrm{p}$ (same): 0.01722).

A Correspondence Analysis was applied to the binary matrix (BM) data (287 individuals x 50 typologies of anomalies, anomaly 12 excluded) to determine whether a different pattern (co- 
occurrence of some anomalies in the two groups) of anomalies existed in the two lots. The very low total variance expressed by the first three Correspondence Axes (14.3\%) and the resulting overlapping ordination model of the two lots in the space defined by the first three correspondence axes indicates the absence of any particular relationship between anomalies and groups, and so the data are not shown.

\section{Discussion}

A non-inflated or abortive swim bladder diminishes the capability of fish to maintain the position in the water column, causing higher swimming activity and the descent of exhausted larvae to the tank bottom, with consequent mortality due to suffocation or infection from the high bacteria loads on the tank bottom, associated with the high density in intensive aquaculture (Woolley and Qin, 2010). However, some fish can survive to the adult stage in aquaculture even without a functional swim bladder, but their inferior quality still remains a major problem in intensive farming. The consequent necessity to cull out juveniles without swim bladder represents an additional cost for the farmer.

The sorting method commonly used by commercial hatcheries is based on the difference in buoyancy between larvae with and without functional swim bladders (Chapman et al., 1988). The efficiency of this sorting method for swim bladder assessment has so far not been convincingly demonstrated (Woolley and Qin, 2012). Conversely, some studies have shown that gases within the swim bladder tend to be released when the larvae are anaesthetised (Massee et al., 1995). In addition, the anaesthesia itself may cause a stress response in some cases (Small, 2003). Therefore, further studies will be necessary to determine the effect of anaesthetics on swim bladder volume during mass selection.

A high incidence of skeletal anomalies in juveniles of gilthead seabream, European seabass, red seabream, yellowtail amberjack and Japanese seabass lacking a functional swim bladder has been observed by many authors (Boglione et al., 1995; Daoulas et al., 1991; Kitajima et al., 1981; Paperna, 1978). In confirmation of these previous publications, this study showed a significantly higher skeletal anomalies load affecting gilthead seabream juveniles lacking a swim bladder. The differences are evidently quantitative because, even if some anomalies typologies were detected only in the WSB lot, their occurrences were so low that Analysis of Correspondences (CA applied to binary data, that is on the presence/absence of each anomaly) failed to find any ordination model capable of separating the two groups on the base of skeletal anomalies.

Some of the results obtained in this study are very different from those hitherto reported in literature. The most significant are the higher anomaly load of the pectoral fin and the absence of 
any clear relationship between abortive swim bladder and axis deviations in the WSB group. This datum contradicts in particular what was previously reported in European seabass (Boglione et al., 1995; Divanach et al., 1997), where lordosis or kyphosis had been associated with abortive swim bladder, but it confirms what is described by Andrades et al. (1996) in seabream. This difference could be attributed to some species-specific features, although the confirmation requires further studies to be carried out on both species using the same methodology.

The consideration that CA was not able to separate the two lots but that the Mann-Whitney U test actually found significant inter-group differences justifies the hypothesis that the observed differences between SB and WSB seabream mainly have a quantitative and not qualitative basis, with the frequency of individuals with anomalous pectoral rays being the more discriminating descriptor between the two lots. No other anomalies were found in any way associated with pectoral fins anomalies, the only exception being the median values of vertebrae number, which was 24 (as in the wild) in WSB seabream without pectoral fin anomalies, and 25 in WSB samples carrying also pectoral fin anomalies. The underlying process is hard to understand at the present level of our knowledge of skeletal processes in fish and only hypotheses can be proposed. In 1978, Paperna described reared gilthead seabream with abortive swim bladder displaying rapid fin strokes, heads pointing upwards, and producing a jerky forward motion to maintain themselves in the upper layer of the water. He observed in the one-year-old seabream with abortive swim bladder extreme kypholordosis, curved and disoriented neural spines and ribs, and deformed pectoral, ventral and caudal fins. In the WSB seabream object of the present study, we observed higher anomaly load on the pectoral fin (associated to higher median value of vertebrae number) and no evident relationship between abortive swim bladder and axis deviations: in facts, some anomalies exclusively checked in WSB seabream (i.e., kyphosis (B2), lordosis (C1), deformed pre-haemal (B4) and haemal (C4) vertebrae, supernumerary vertebral arches $\left(\mathrm{C} 5^{*}, \mathrm{C6}^{*}\right)$, supernumerary radial (E23R) or pterygophore (H23), defective rays (F11, L11R), urinary calculi (13) and mineralization loss in epurals (20)), were quite rare (0.4-3.8\% of individuals). No kypholordosis was detected. Anomalous rays in all (with the exception of pectoral rays: F-I11) fins and anomalous neural elements (A-D5) affected the same proportion of seabream in both groups. The fact that no relationship between swim bladder and axis anomalies was found could be due to many different factors. Paperna, for instance, did not describe the skeletal anomalies present in seabream with normal swim bladder, so it is not possible so far to know which anomalies were actually exclusive of the WSB seabream. Other studies (Andrades et al., 1996) analyzed at a greater detail a reduced number (a total of 50 normal and lordotic sea bream) of X-rays of larger fishes, so no data can be used for a comparison. The different sizes (juveniles vs sub-adult or adult) of seabream analysed in the different studies 
could be the main reason for the diversity found in the skeletal anomalies associated with abortive swim bladder, with the possibility that some time (growth) is necessary before the effects of abortive swim bladder on vertebral axis are detectable. Under this hypothesis, skeletal anomalies related to abortive swim bladder might not yet be fully expressed in the young stages we analysed in this study. It could be hypothesised that an overuse of pectoral fin flapping (i.e., for rapid fin strokes) is established by larvae with abortive swim bladder in order to maintain the desired level in the water column. This could increase the activity of pectoral muscles in the first ontogenetic period, augmenting the mechanical load on the surrounding muscles and bones. In this scenario, the doubled ratio of pectoral fins anomalies observed in WSB seabream may be the consequence of an augmented pressure exerted by pectoral muscles, according to Thorsen and Westneat (2005), that described the pectoral fin in fish involved in fore-aft rowing stroke used for high transient thrust and manoeuvrability.

Loss of mineralization and the presence of supernumerary elements in pectoral fins, detected only in the WSB lot, could thus be the consequence of altered mineralization/transdifferentiation processes caused by an extra load on differentiating pectoral fin. The same release of a more intense mechanical load on ossifying pre-haemal vertebrae (which are located over the pectoral fin) may cause some gradual bending of notochord and vertebral axis, with shape anomaly and fusion of prehaemal vertebrae (Boglione et., 1995; Chatain, 1982; Kitajima, 1978) at first, later extending to the haemal vertebrae. On the other hand, it is ascertained that increased compressive load influence skeletal development, modelling and remodelling, i.e., extra-load exercised by hypertrophic muscle on underlying vertebrae (Kranenbarg et al., 2005b; Fjeldall et al., 2012; Totland et al., 2011) provoke vertebral anomalies in reared salmons; high mechanical load induces cartilage (Hall and Witten, 2007) in the vertebral column of many teleost species (Kranenbarg et al., 2005a,b; Witten et al., 2005); Hall $(1973$; 1968) postulated that adventitious cartilage arises in response to intermittent pressure and tension accompanied by motion. Endochondral ossification during skeletal morphogenesis is regulated to a greater extent by the mechanical loading histories: the normal sequence of events for cartilage in the appendicular skeleton (proliferation, maturation, degeneration, and ossification) is accelerated by intermittent shear stresses and inhibited or prevented by intermittent hydrostatic compressive stresses, according to Pauwels (1980).

Other environmental factors can be however implicated in inducing skeletal anomalies in gilthead seabream with abortive swim bladder, i.e., in conditions of increased workload, locomotory muscles in rainbow trout (Oncorhynchus mykiss) extract a greater proportion of oxygen from the blood, in this way the oxygen content in the venous blood decreases (Farrell and Clutterham, 2003). Carter et al. (1988) outlined how in much vertebrate skeletal development, osteogenic precursor cells in 
regions of low oxygen tension tend to be shunted into a chondrogenic rather than an osteogenic pathway. Even temperatures differing from the species specific one during the hatchery rearing phase could affect oxygen consumption and consequently the swimming capacity: in adult sockeye (Oncorhynchus nerka) and coho (O. kisutch) salmon stocks, Lee et al. (2003) found that the relationship between water temperature and maximum oxygen consumption $\left(\dot{M}_{\mathrm{O} 2 \mathrm{max}}\right)$, i.e. the $\dot{M}_{\mathrm{O} 2}$ measured at the critical swimming speed $\left(U_{\text {crit }}\right)$, revealed temperature optima for $\dot{M}_{\mathrm{O} 2 \max }$ that were stock-specific.

This scenario is in good agreement with some defects we observed in pectoral fins (reduced radials, loss of mineralization in radials).

In conclusion, the fact that we did not observe any convincing relationship between abortive swim bladder and axis deviation in the haemal region could be due to the early stage at which we carried out our observations. However, this study is the first that describes skeletal anomalies and meristic counts in gilthead seabream without an inflated swim bladder at a given age and length, thus representing a basis for further investigations. On the basis of our results, the hypothesis that mechanical extra load exerted by muscles on differentiating pectoral fin at first, pre-haemal vertebrae later, to compensate for the lack of a functional swim bladder, was formulated to explain the relationship observed between abortive swim bladder and pectoral fins (and rarer pre-haemal vertebrae) anomalies found in this study, and the presence of axis anomalies described in older stages in literature.

External mechanical forces are recognized as regulating genetic pathways of both cartilage and bone development in all vertebrates, in this way providing the skeletal tissues with the capability to adapt their structures, shape and mechanical features in response to altered loading conditions in teleost fish (Fiaz et al., 2012; Huysseune et al., 1994; Kranenbarg et al., 2005a,b). Currently, a large body of studies deals with mechano-regulated tissue differentiation models developed for humans according to Pauwels's theory (Pauwels, 1980), where the mechanical environment in the medium is assumed to determine tissue phenotypes. Further and deeper investigations are still necessary in fish at cellular level to definitely prove this hypothesisfor other species and/or life stages.

\section{Acknowledgement}

This study was funded by the Italian Ministry for Agriculture, Food and Forestry Policy (Law 41/82 Project n. 5c49 - V Three-Year Plan). It does not necessarily reflect the view of the Italian Ministry for Agriculture, Food and Forestry Policy or the European Commission. It in no way anticipates the Commission's future policy in this area. The funders had no role in study design, data collection 
and analysis, decision to publish, or preparation of the manuscript.

This study was conducted in the context of the PhD study by the first author.

The authors would like to thank Dr. Attilio Spanò (Civita Ittica s.r.l., Civitavecchia Rome, Italy) for the samples offered for this research and two anonymous Referees that greatly contributed to ameliorate this paper.

\section{References}

Andrades, J.A., Becerra, J., Fernandez-Llebrez, P., 1996. Skeletal deformities in larval juvenile and adult stages of cultured gilthead sea bream (Sparus aurata L.). Aquaculture 141, 1-11.

Battaglene, S.C., Talbot, R.B., 1990. Initial swim bladder inflation in intensively reared Australian bass larvae, Macquaria novemaculeata (Steindachner) (Perciformes: Percichthyidae). Aquaculture $36,431-442$.

Bennet, R.O., Kraeuter, J.N., Woods, L.C., Lipsky, M.M., May, E.B., 1987. Histological evaluation of swim bladder non-inflation in striped bass larvae, Morone sexatilis. Dis. Aquat. Org. 3, 91-95.

Benzecri, J.P., 1973. L'Analyse des Données. L'Analyse des Correspondances, vol. 2. Dunod, Paris, France, pp. 628.

Boglione, C., Costa, C., Di Dato, P., Ferzini, G., Scardi, M., Cataudella, S., 2003. Skeletal quality assessment of reared and wild sharpsnout sea bream and pandora juveniles. Aquaculture 227, 373394.

Boglione, C., Costa, C., Giganti, M., Di Dato, P., Scardi, M., Cataudella, S., 2006. Biological monitoring of wild thicklip grey mullet (Chelon labrosus), golden grey mullet (Liza aurata), thinlip mullet (Liza ramada) and flathead mullet (Mugil cephalus) (Pisces: Mugilidae) from different Adriatic sites: Meristic counts and skeletal anomalies. Ecol Indic 6, 712-732.

Boglione, C., Gagliardi, F., Scardi, M., Cataudella, S., 2001. Skeletal descriptors and quality assessment in larvae and post-larvae of wild-cought and hatchery-reared gilthead sea bream (Sparus aurata L. 1758). Aquaculture 192, 1-22.

Boglione, C., Marino, G., Fusari, A., Ferreri, F., Finoia, M.G., Cataudella, S., 1995. Skeletal anomalies in Dicentrarchus labrax juveniles selected for functional swimbladder. ICES marine Science Symposium 201, 163-169.

Boglione, C., Marino, G., Giganti, M., Longobardi, A., De Marzi, P., Cataudella, S., 2009. Skeletal anomalies in dusky grouper Epinephelus marginatus (Lowe 1834) juveniles reared with different methodologies and larval densities. Aquaculture 291, 48-60.

Bulak, J.S., Heidinger, R.C., 1980. Developmental anatomy and inflation of the swim bladder in striped bass, Morone sexatilis. Fish. Bull. 77(4), 1000-1002.

Carter, D.R., Blenman, P.R., Beauprk, G.S., 1988. Correlations between Mechanical Stress History and Tissue Differentiation in Initial Fracture Healing Journal of Orthopaedic Research 6736-748, Raven Press, Ltd., New York Orthopaedic Research Society 
Cataudella, S., Russo, T., Lubrano, P., De Marzi, P., Spanò, A., Fusari, A., Boglione, C., 2002. An ecological approach to produce "wild like"' juveniles of sea bass and sea bream: trophic ecology in semi-intensive hatchery conditions. Aquaculture Europe 2002 Conference, Trieste, Italy, October 16-19, 2002, European Aquaculture Society, Extended Abstracts and Short Communications, Special Publication no. 32, Oostende, Belgium, pp. 177-178.

Chapman, D.C., Hubert, W.A., 1988. Influence of access to air and of salinity on gas bladder inflation in striped bass. Prog. Fish.-Cult. 50, 23-2.

Chatain, B., 1982. Contribution à l'étude de l'éelevage larvaire de la dorade japonaise (Chrysophrys major). Thèse de 3e cycle, Universitaté s’Aix-Marseille II, France, p. 121.

Chatain, B., 1986. La vessie natatoire chez Dicentrarchus labrax et Sparus aurata. I. Aspects morphologiques du développement. Aquaculture 53, 303-311.

Chatain B., 1989. Problems related to the lack of swimbladder in intensive rearing of Dicentrarchus labrax et Sparus auratus. Advances in tropical aquaculture, Aquacop. IFRE-MER. Actes de colloque, 9 (1989), pp. 699-709 Tahiti, Feb. 20-March 4, 1989.

Chatain, B., 1994. Abnormal swimbladder development and lordosis in sea bass (Dicentrachus labrax) and sea bream (Sparus aurata). Aquaculture 97, 169-180.

Chatain, B., Ounais-Guschemann, N., 1990. Improved rate of initial swimbladder inflation in intensively reared Sparus aurata. Aquaculture 84, 345-353.

Daoulas, Ch., Economou, A. N., Bantavas, I., 1991. Osteological abnormalities in laboratory reared sea-bass (Dicentrarchus labrax) fingerlings. Aquaculture 97, 169-180.

Dingerkus, G., Uhler, L.D., 1977. Enzyme clearing of alcian blue stained whole small vertebrates for demonstration of cartilage. Stain. Techn. 52(4), 229-232.

Divanach, P., Papandroulakis, N., Anastasiadis, P., Koumoundouros, G., Kentouri, m., 1997. Effect of water currents on the development of skeletal deformities in sea bass (Dicentrarchus labrax L.) with functional swimbladder during postlarval and nursery phase. Aquaculture 156, 145-155.

Doroshev, S.I., Cornacchia, J.W., 1979. Initial swimbladder inflation in the larvae of Tilapia mossambica (Peters) and Morone sexatilis (Walbaum). Aquaculture 16, 57-66.

Farrell, A.P., Clutterham, S.M., 2003. On-line venous oxygen tensions in rainbow trout during graded exercise at two acclimation temperatures. J. Exp. Biol. 206, 487-496.

Fiaz, A.W., Leon-Kloosterziel, K.M., Gort, G., Schulte-Merker, S., van Leeuwen, J.L., Kranenbarg, S., 2012. Swim-training changes the spatio-temporal dynamics of skeletogenesis in zebrafish larvae (Danio rerio). PLoS ONE 7(4), e34072. doi:10.1371/journal.pone.0034072.

Fielder, D.S., Bardsley, W.J., Allan, G.L., Pankhurst, P.M., 2002. Effect of photoperiod on growth and survival of snapper Pagrus auratus larvae. Aquaculture 211, 135-150. 
Fjelldal, P.G., Hansen, T., Breck, O., Ørnsrud, R., Lock, E.-J., Waagbø, R., Wargelius, A. Witten, P.E., 2012. Vertebral deformities in farmed Atlantic salmon (Salmo salar L.) - etiology and pathology Journal of Applied Ichthyology 28(3), 433-440. DOI:10.1111/j.1439-0426.2012.01980.x

Giavenni, R., Doimi, M., 1983. Formazione, differenziamento istologico ed aspetti patologici della vescica natatoria in larve di branzino (Dicentrarchus labrax L., 1758). Riv. It. Piscic. Ittiop. A. XVIII 2, 71-80.

Hall, B.K., 1968. In vitro studies on the mechanical evocation of adventitious cartilage in the chick. J. Exp. Zoo1. 168, 283-306,

Hall B.K.1973. Immobilization and cartilage transformation into bone in the embryonic chick. Anat. Rec. 173, 391-404.

Hall, B.K. Witten, P.E., 2007. The origin and plasticity of skeletal tissues in vertebrate evolution and development, in: Anderson, J.S., Sues, H.D. (Eds), Major transitions in vertebrate evolution. and. Indiana University Press, Bloomington, IN, pp. 432.

Hammer, Ø., Harper, D. A. T., Ryan, P. D., 2001. PAST: Paleontological Statistics Software Package for Education and Data Analysis. Palaeontologia Electronica 4, p. 9.

Harder, W., 1975. Anatomy of Fishes. Part I., E Schweizebart'sche Verlagsbuchhandlung (Nagele u Obermiller), Stuttgart. p. 612.

Huysseune, A., Sire, J.Y., Meunier, F.J., 1994. Comparative study of lower pharyngeal jaw structure in two phenotypes of Astatoreochromis alluaudi (Teleostei: Cichlidae). Journal of Morphology 221, 2-43.

Iseda, H., Ishihara, M., Sumida, S., Owaki, M., Tabata, S., 1979. Prevention of deformation in the juveniles of red sea bream, Pagrus major, reared in ponds. Relationship between the initial rearing conditions and the lordotic deformity. Bull. Kumamoto Pref. Fish. Exp. Stn. 1, 9-17.

Kitajima, C., 1978. Acquisition of fertilized eggs and mass culture of juveniles of red sea bream, Pagrus major. Spec. Rep. 5, Nagasaki Prefecture Institute of Fisheries, p. 92.

Kitajima, C., Iwamoto, H., Fuhita, S., 1977. Relation between curvature of vertebral column and hatchery-reared undeveloped swim bladder in red sea bream Pagrus major. Bull. Nagasaki Pref. Inst. Fish. 3, 23-32.

Kitajima, C., Tsukashima, Y., Fujita, S., Watanabe, T., Yone, Y., 1981. Relationship between uninflated swimbladder and lordotic deformity in hatchery-reared red sea bream, Pagrus major. Bulletin of the Japanese Society of Scientific Fisheries 47, 1289-1297.

Kohno, H., Taki, Y., Ogasawara, Y., Shirojo, Y., Taketomi, M., Inoue, M., 1983. Development of swimming and feeding functions in larval Pagrus major. Jap. J. Icht. 30, 47-59. 
Kranenbarg, S., Waarsing, J.H., Muller, M., Weinans, H., van Leeuwen, J.L., 2005a. Adaptive bone formation in acellular vertebrae of sea bass (Dicentrarchus labrax L.). Journal of Biomechanics 38, 3493-3502.

Kranenbarg, S., Waarsing, J.H., Muller, M., Weinans, H., van Leeuwen, J.L., 2005b. Lordotic vertebrae in sea bass (Dicentrarchus labrax L.) are adapted to increased loads. Journal of Biomechanics 38, 1239-01246.

Lee, C.G., Farrell, A.P., Lotto, A., MacNutt, M.J., Hinch, S.G, Healey, M.C., 2003. The effect of temperature on swimming performance and oxygen consumption in adult sockeye (Oncorhynchus nerka) and coho (O. kisutch) salmon stocks. J. Exp. Biol. 206, 3239-3251.

Marino, G., Boglione, C., 1990. Contributo allo studio delle anomalie più comuni in stadi larvali di spigola (Dicentrarchus labrax) ottenuti per riproduzione controllata. Atti S.I.S.V.E.T. vol. XLIV, 1055-1062.

Marty, G.D., Hinton, D.E., Summerfelt, R.C., 1995. Histopathology of swim bladder non-inflation in walleye (Stizostedion vitreum) larvae: role of development and inflammation. Aquaculture 138, $35-48$.

Massee, K.C., Rust, M.B., Hardy, R.W., Stickney, R.R., 1995. The effectiveness of tricaine, quinaldine sulfate and metomidate as anesthetics for larval fish. Aquaculture 134, 351-359.

Matsuoka, M., 1987. Developmental of skeletal tissues and skeletal muscles in the red sea bream. Bull. Seikai Reg. Fish. Res. Lab. 65, 1-114.

Modica, A., Santulli, A, Curatolo, A., Cusenza, L., Palillo, L., D’Amelio, V., 1993. Relatioship between absence of functional swimbladder, calculosis and larval mortality in hatchery-reared gilthead sea bream, Sparus aurata L. Aquacult. Fish. Mgt. 24, 517-522.

Nash, C.E., Kuo, C.M., Madden, W.D., Paulsen, C.L., 1977. Swim bladder inflation and survival of Mugil cephalus to 50 days. Aquaculture 12, 89-94.

Paperna, I., 1978. Swimbladder and skeletal deformations in hatchery bred Sparus aurata. Journal of Fish Biology 12, 109-114.

Pauwels, F., 1980. Biomechanics of the Locomotor Apparatus, $1^{\text {st }}$ edn. Springer-Verlag, Berlin, Heidelberg, New York, NY.

Peruzzi, S., Westgaard, J.I., Chatain, B., 2007. Genetic investigation of swimbladder inftation anomalies in the European sea bass, Dicentrarchus labrax L. Aquaculture 265(1-4), 102-108.

Prestinicola, L., Boglione, C., Makridis, P., Spanò, A., Rimatori, V., Palamara, E., Scardi, M., Cataudella, S., 2013. Environmental Conditioning of Skeletal Anomalies Typology and Frequency in Gilthead Seabream (Sparus aurata L., 1758) Juveniles. PLoS ONE 8(2), e55736. doi: 10.1371/journal.pone.0055736. 
Schultze, H.P., Arratia, G., 1989. The composition of the caudal skeleton of Teleosts

Small, B.C., 2003. Anesthetic efficacy of metomidate and comparison of plasma cortisol responses to tricaine methanesulfonate, quinaldine and clove oil anesthetized channel catfish Ictalurus punctatus. Aquaculture 217, 177-185.

Thorsen, D.H., Westneat, M.W., 2005. Diversity of Pectoral Fin Structure and Function in Fishes With Labriform Propulsion. Journal of Morphology 263,133-150.

Totland, G.K., Fjelldal, P.G., Kryvi, H., Løkka, G., Wargelius, A., Sagstad, A., Hansen, T., Grotmol, S., 2011. Sustained swimming increases the mineral content and osteocyte density of salmon vertebral bone. J. Anat. 219, 490-501.

Trotter A.J., Pankhurst P.M., Hart P.R., 2001. Swim bladder malformation in hatchery-reared striped trumpeter Latris lineate (Latridae). Aquaculture 198, 41-54.

Trotter, A.J., Battaglene, S.C., Pankhurst, P.M., 2003. Effects of photoperiod and light intensity on initial swim bladder inflation, growth and post-inflation viability in cultured striped trumpeter (Latris lineata) larvae. Aquaculture 224, 141-158.

Trotter, A.J., Pankhurst, P.M., Battaglene, S.C., 2005. A finite interval of initial swim bladder inflation in Latris lineata revealed by sequential removal of water surface films. Journal of Fish Biology 67, 730-741.

Watanabe, Y., Wei, Q., Yang, D., Chen, X., Du, H., Yang, J. Sato, K., Naito, Y., Miyazaki, N., 2008. Swimming behavior in relation to buoyancy in an open swimbladder fish, the Chinese sturgeon. Journal of Zoology 275, 381-390.

Weppe, M., Bonami, J.R., 1983. Non-inflation of the swimbladder in hatchery-reared sea bass and sea bream: a significant problem in marine aquaculture. Bull. Eur. Ass. Fish Pathol. 3(4), 59-60.

Witten,P.E., Gil-Martens, L., Hall, B.K., Huysseune, A., Obach, A., 2005. Compressed vertebrae in Atlantic salmon Salmo salar: evidence for metaplastic chondrogenesis as a skeletogenic response late in ontogeny. Dis Aquat Org. 64, 237-246.

Woolley, L.D., Qin, J.G., 2010. Swimbladder inflation and its implication to the culture of marine finfish larvae. Reviews in Aquaculture 2, 181-190.

Yamaoka, K., Nambu, T., Miyagawa, M., Isshiki, T., Kusaka, A., 2000. Water surface tensionrelated deaths in prelarval redspotted grouper. Aquaculture 189, 165-176. 
Table 1: Characteristics of the reared gilthead seabream lots. $n=$ number of individuals; $S L=$ standard length; DAH = days after hatching.

\begin{tabular}{ccl}
\hline Code & n & \multicolumn{1}{c}{ Characteristics } \\
\hline SB & 50 & $\begin{array}{l}\text { Seabream juveniles with inflated swim bladder sampled at 109 DAH. Mean } \\
\text { SL: 23.9 mm; Range SL: 16.0-40.0 mm. }\end{array}$ \\
WSB & 237 & $\begin{array}{l}\text { Seabream juveniles with uninflated swim bladder sampled at 109 DAH. Mean } \\
\text { SL: 24.5 mm; Range SL: 17.0-47.0 mm. }\end{array}$ \\
\hline Total & $\mathbf{2 8 7}$ & \\
\hline
\end{tabular}


Table 2: List of considered anomalies. Italics highlight the severe anomalies, defined as those that affect the external shape of the fish body and impair fish performance.

\begin{tabular}{|c|c|c|}
\hline & Code & Description \\
\hline \multirow{10}{*}{ 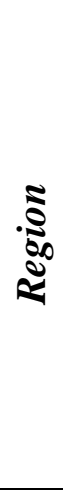 } & A & Cephalic vertebrae (carrying epipleural ribs) \\
\hline & B & Pre-haemal vertebrae (carrying epipleural, pleural ribs and open haemal arch, without \\
\hline & $\mathrm{C}$ & Haemal vertebrae (with haemal arch closed by haemal spine) \\
\hline & $\mathrm{D}$ & Caudal vertebrae (with haemal and neural arches closed by modified spines) \\
\hline & $\mathrm{E}$ & Pectoral fin \\
\hline & $\mathrm{F}$ & Anal fin \\
\hline & G & Caudal fin \\
\hline & $\mathrm{H}$ & Dorsal spines \\
\hline & I & Dorsal soft rays \\
\hline & $\mathrm{L}$ & Pelvic fin \\
\hline \multirow{34}{*}{$\begin{array}{l}\frac{0}{80} \\
\frac{8}{8} \\
\frac{8}{8}\end{array}$} & $\mathrm{~S}$ & Scoliosis \\
\hline & SBS & Saddle-back \\
\hline & 1 & Kyphosis \\
\hline & 2 & Lordosis \\
\hline & 3 & Partial vertebral fusion \\
\hline & $3 *$ & Total vertebral body fusion \\
\hline & 4 & $\begin{array}{l}\text { Vertebral body anomaly (abnormally shaped, ossification ridges, marked reduction in } \\
\text { length or elongation) }\end{array}$ \\
\hline & 5 & Anomalous (abnormally shaped, twisted, reduced, fused) neural arch and/or spine \\
\hline & $5^{*}$ & Supernumerary or absent neural elements of vertebrae \\
\hline & 6 & Anomalous (abnormally shaped, twisted, reduced, fused) haemal arch and/or spine \\
\hline & $6^{*}$ & Supernumerary or absent haemal elements of vertebrae \\
\hline & 7 & Anomalous (abnormally shaped) or supernumerary rib \\
\hline & 8 & $\begin{array}{l}\text { Anomalous pterygophores/radial (abnormally shaped, twisted, absent, fused, } \\
\text { supernumerary) }\end{array}$ \\
\hline & 9 & Anomalous hypural (absent, fused, supernumerary) \\
\hline & $9 *$ & Anomalous or broken parahypural or fused with hypurals or haemaspine \\
\hline & 10 & Anomalous epural (abnormally shaped, absent, fused, supernumerary) \\
\hline & 11 & Anomalous ray (absent, fused, supernumerary, shorter, longer, twisted) \\
\hline & 12 & Uninflated swim-bladder \\
\hline & 13 & Presence of calculi in the urinary ducts \\
\hline & 14 & Anomalous (reduced, deformed, protruding) maxillary and/or pre-maxillary \\
\hline & 15 & Anomalous (reduced, deformed, protruding) dentary \\
\hline & 16 & Other cephalic anomalies (glossohyal, neurocranium, ..) \\
\hline & $17 L / R$ & Anomalous left/right opercular plate \\
\hline & $17 * \mathrm{~L} / \mathrm{R}$ & Anomalous, absent, fused branchiostegal ray \\
\hline & 18 & Abnormally shaped, twisted, reduced, fused predorsal bones \\
\hline & 19 & Hypural with mineralization defect \\
\hline & 20 & Epural with mineralization defect \\
\hline & 21 & Pterygophore/radial with mineralization defect \\
\hline & $22 \mathrm{~L} / \mathrm{R}$ & Left/right opercular plate with mineralization defect \\
\hline & 23 & Supernumerary bone \\
\hline & 24 & Urostyle with mineralization defect \\
\hline & 25 & Vertebral bodies with mineralization anomalies (hyperdense, loss of mineralization) \\
\hline & 26 & Abnormally shaped postcleithrum \\
\hline & 27 & Abnormally shaped left/right cleithrum \\
\hline
\end{tabular}


Table 3: Characteristics of wild gilthead seabream lots, belonging to the LESA historical dataset, here used as terms of reference. $n=$ number of individuals; $S L=$ standard length.

\begin{tabular}{|c|c|c|}
\hline Lot & n & Characteristics \\
\hline WIIT01 & 72 & $\begin{array}{l}\text { Wild juveniles (Adriatic sea); average LS: } 19.9 \mathrm{~mm} \text {; SL range: } 9.5-49 \\
\text { mm. }\end{array}$ \\
\hline WIIT02 & 41 & $\begin{array}{l}\text { Wild juveniles sampled near Chioggia (Adriatic sea); average SL: } 38.1 \\
\text { mm; SL range: } 25-43 \mathrm{~mm} \text {. }\end{array}$ \\
\hline WIIT03 & 60 & $\begin{array}{l}\text { Wild juveniles sampled near Chioggia (Adriatic sea); average SL: } 58.1 \\
\mathrm{~mm} \text {; SL range: } 52-70 \mathrm{~mm} \text {. }\end{array}$ \\
\hline WIIT04 & 16 & Wild juveniles (Adriatic sea); average SL: $20 \mathrm{~mm}$. \\
\hline WIIT05 & 149 & $\begin{array}{l}\text { Same wild lot as WIIT04 (subadult); average SL: } 146.4 \mathrm{~mm} \text {; SL range: } \\
130-168 \mathrm{~mm}\end{array}$ \\
\hline WITU01 & 88 & $\begin{array}{l}\text { Wild juveniles from Aegean sea, off Turkish coast; average LS: } 19.8 \mathrm{~mm} \text {; } \\
\text { LS range: } 11.5-44 \mathrm{~mm} \text {. }\end{array}$ \\
\hline total & 426 & \\
\hline
\end{tabular}


Table 4: Results of meristic counts: ranges of values observed in reared and wild lots. Bold indicates values out of the range observed in wild seabream.

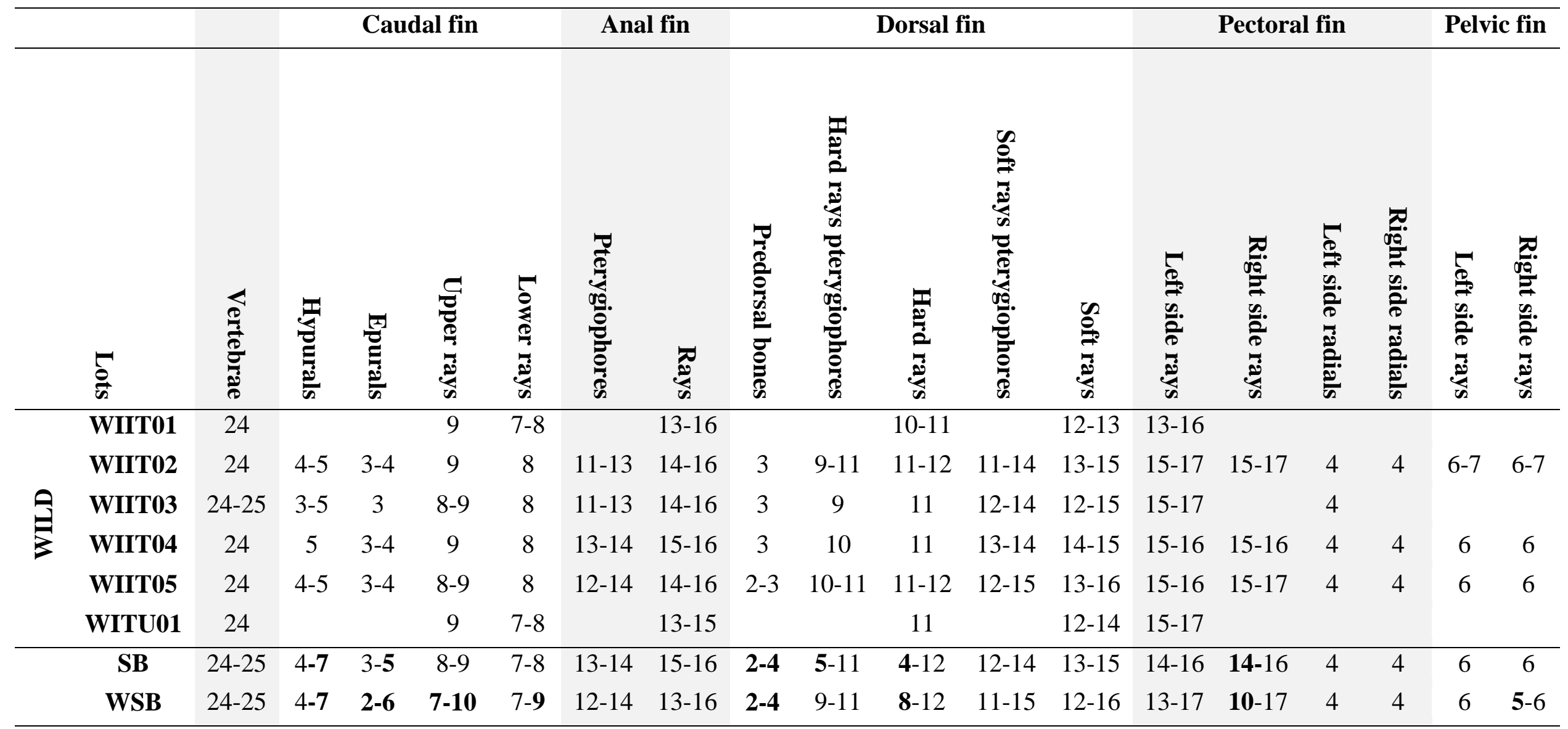


Table 5: Results of skeletal anomalies analysis: general data. *=values obtained by excluding the anomaly 12 (absence of swim bladder) from calculation.

\begin{tabular}{lccc}
\hline & SB & WSB & WSB* \\
\hline N. individuals observed & 50 & 237 & 237 \\
Total frequency $(\%)$ of anomalous individuals & 100.0 & 100.0 & 100.0 \\
Average anomalies load & 8.8 & 10.8 & 9.8 \\
Frequency individuals with at least one severe anomaly $(\boldsymbol{\%})$ & 42.0 & 100.0 & 48.5 \\
Frequency of severe anomalies out of the total $(\boldsymbol{\%})$ & 7.8 & 17.2 & 8.7 \\
Severe anomalies load & 1.6 & 1.8 & 1.7 \\
\hline
\end{tabular}


Table 6: Relative frequencies (\%) of each anomaly typology and of individuals affected by each anomaly typology, in each lot. No value means $0.0 \%$. Bold codes highlight severe anomalies.

Italics indicate typologies with the highest frequencies of occurrence. Boxes highlight typologies occurring only in seabream with uninflated swim bladder.

Anomaly Frequency $(\%)$ of each anomaly typology

\begin{tabular}{|c|c|c|c|c|}
\hline & SB & WSB & SB & WSB \\
\hline $\mathbf{S}$ & & 0.2 & & 2.5 \\
\hline SDB & 0.2 & 0.2 & 2.0 & 1.7 \\
\hline A5 & \multirow[t]{3}{*}{2.7} & 1.5 & \multirow[t]{3}{*}{14.0} & 13.9 \\
\hline B2 & & 0.1 & & 0.8 \\
\hline B4 & & 0.1 & & 0.8 \\
\hline$B 5$ & \multirow[t]{4}{*}{13.0} & 9.5 & \multirow[t]{4}{*}{50.0} & 40.5 \\
\hline B7left & & 0.2 & & 1.7 \\
\hline C1 & & 0.1 & & 0.8 \\
\hline C4 & & 0.5 & & 3.8 \\
\hline$C 5$ & \multirow[t]{2}{*}{15.5} & 14.6 & \multirow[t]{2}{*}{58.0} & 57.0 \\
\hline C5* & & 0.1 & & 0.4 \\
\hline C6 & \multirow[t]{2}{*}{14.6} & 13.9 & \multirow[t]{2}{*}{52.0} & 62.9 \\
\hline C6* & & 0.2 & & 1.7 \\
\hline D3 & 0.2 & 0.5 & 2.0 & 5.1 \\
\hline D3* & 0.9 & 0.7 & 8.0 & 8.0 \\
\hline D4 & 3.4 & 3.0 & 14.0 & 15.2 \\
\hline D5 & 1.1 & 1.3 & 10.0 & 11.4 \\
\hline D5* & 0.5 & 0.2 & 4.0 & 1.7 \\
\hline D6 & 3.6 & 3.1 & 30.0 & 26.2 \\
\hline D6* & 0.7 & 0.5 & 6.0 & 5.9 \\
\hline D23 & 0.2 & 0.1 & 2.0 & 0.8 \\
\hline E8L & 0.5 & 0.9 & 4.0 & 8.9 \\
\hline E8R & 0.9 & 0.5 & 8.0 & 5.9 \\
\hline$E 11 L$ & 3.9 & 4.6 & 14.0 & 28.7 \\
\hline$E 11 R$ & \multirow[t]{2}{*}{3.0} & 4.7 & \multirow[t]{2}{*}{16.0} & 22.8 \\
\hline E21L & & 0.3 & & 2.1 \\
\hline E21R & \multirow[t]{2}{*}{0.2} & 0.2 & \multirow[t]{2}{*}{2.0} & 1.7 \\
\hline E23R & & 0.03 & & 0.4 \\
\hline F8 & \multirow[t]{2}{*}{0.2} & 0.2 & \multirow[t]{2}{*}{2.0} & 1.7 \\
\hline F11 & & 0.1 & & 0.8 \\
\hline G9 & 5.2 & 4.3 & 40.0 & 39.2 \\
\hline G9* & 1.4 & 1.2 & 12.0 & 12.7 \\
\hline G10 & 10.7 & 8.6 & 72.0 & 68.4 \\
\hline G11 & 2.7 & 2.0 & 12.0 & 13.1 \\
\hline$G 23$ & 5.2 & 4.1 & 44.0 & 43.5 \\
\hline $\mathrm{H} 8$ & 0.5 & 0.3 & 2.0 & 2.5 \\
\hline H11 & \multirow[t]{2}{*}{0.5} & 0.5 & \multirow[t]{2}{*}{4.0} & 5.5 \\
\hline $\mathrm{H} 23$ & & 0.2 & & 1.7 \\
\hline I8 & 0.2 & 0.4 & 2.0 & 3.4 \\
\hline I11 & 2.5 & 2.5 & 14.0 & 13.5 \\
\hline L11L & \multirow[t]{4}{*}{0.2} & 0.2 & \multirow[t]{4}{*}{2.0} & 1.7 \\
\hline L11R & & 0.1 & & 0.8 \\
\hline 12 & & 9.3 & & 100.0 \\
\hline 13 & & 0.1 & & 1.3 \\
\hline 14 & 0.5 & 0.4 & 4.0 & 4.6 \\
\hline 15 & 0.2 & 0.2 & 2.0 & 2.1 \\
\hline 17L & 1.1 & 0.9 & 10.0 & 10.1 \\
\hline $17 R$ & 1.1 & 0.9 & 10.0 & 9.3 \\
\hline 18 & 1.6 & 1.1 & 14.0 & 12.2 \\
\hline 19 & \multirow[t]{2}{*}{0.7} & 0.7 & \multirow[t]{2}{*}{6.0} & 7.6 \\
\hline 20 & & 0.2 & & 2.5 \\
\hline
\end{tabular}

Frequency of individuals affected by each anomaly typology 


\section{Legend}

Fig. 1: Pectoral fin anomalies inspected in reared gilthead seabream with uninflated swim bladder. A: Normal pectoral fin radials; B: fused and deformed radials. C: the first radial (*) is vestigial (shorter) and a supernumerary radial (arrowhead) is present; D: deformed pectoral rays (arrowheads); E: deformed $4^{\text {th }}$ pectoral radial, and loss of mineralization in the $3^{\text {rd }}$ and $4^{\text {th }}$ ones (arrowheads).

Fig. 2: Examples of mineralization anomalies. A: Loss of mineralization in hypural 3 (arrow) and fusion underway between the $2^{\text {nd }}$ and the $3^{\text {rd }}$ ones (arrowheads); B: more advanced stage of loss of mineralization in the third hypural. 
Fig. 1: Pectoral fin anomalies inspected in reared Gilthead sea bream with uninflated swim bladder.

A
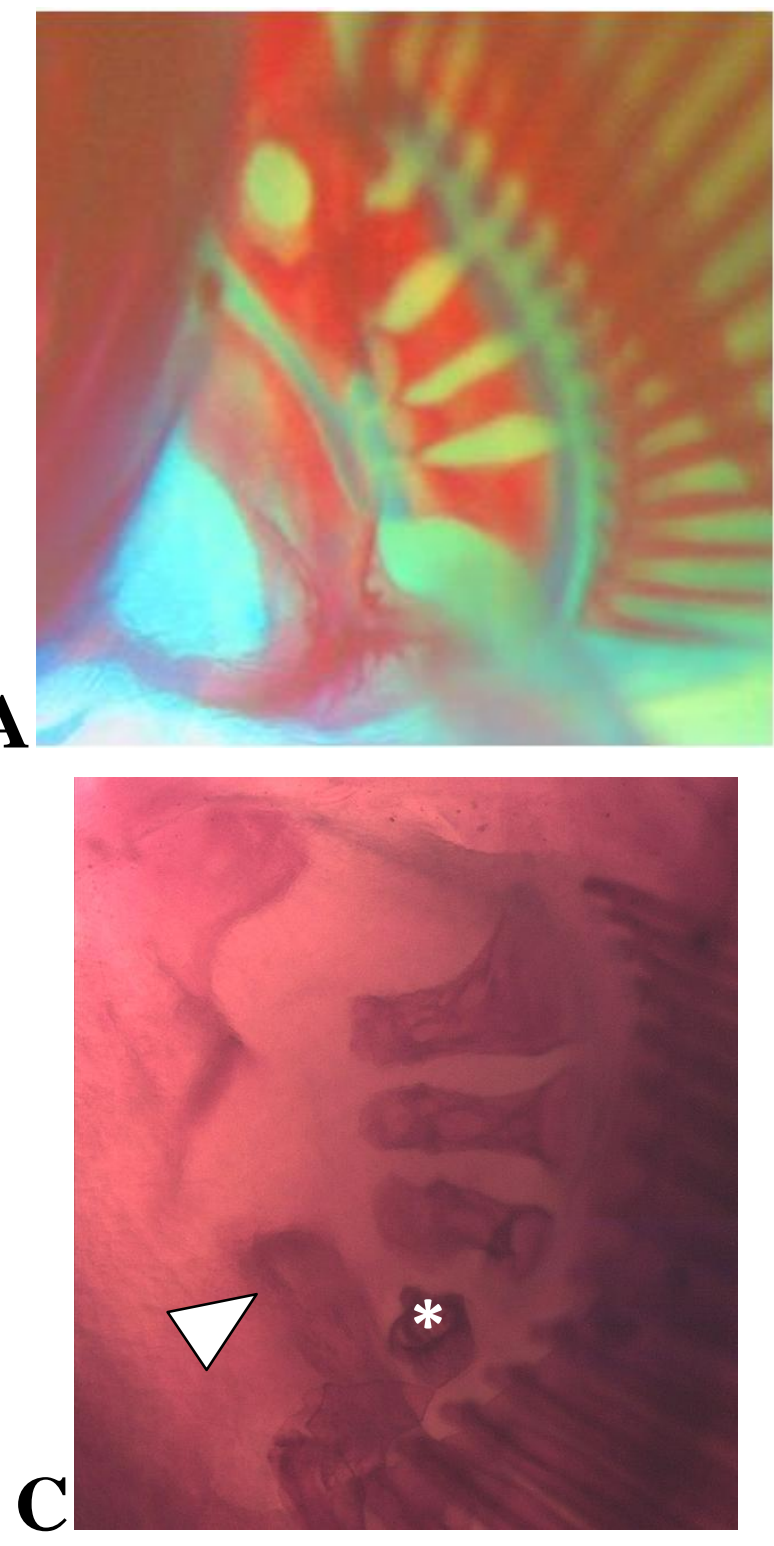

$\Delta$

$\triangle$

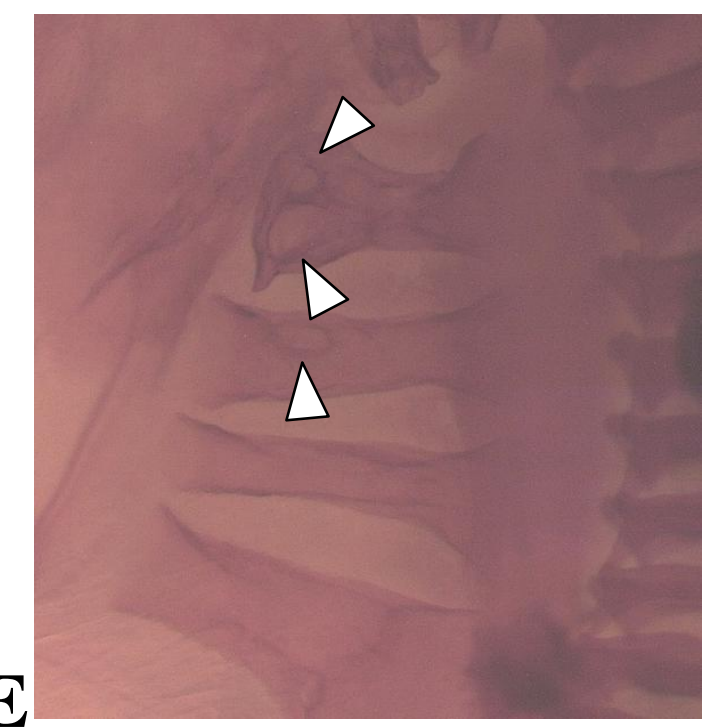

B
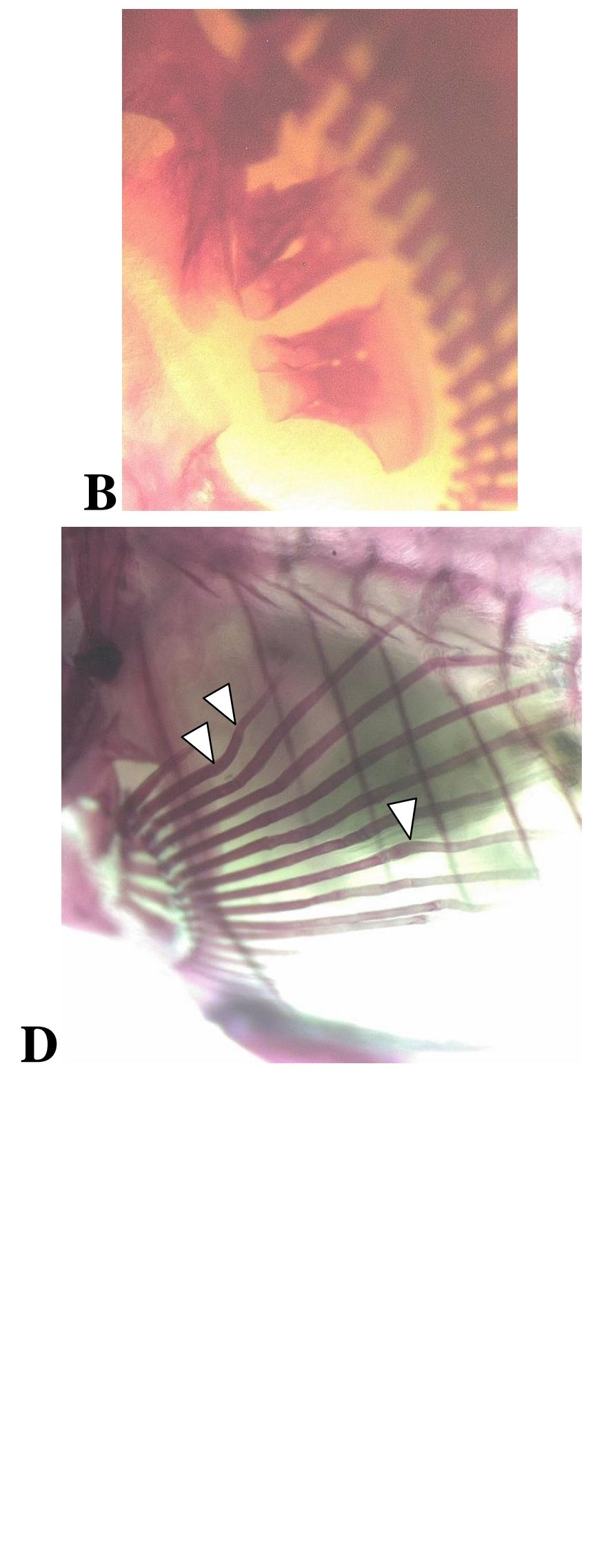
Fig. 2: Examples of mineralization anomalies

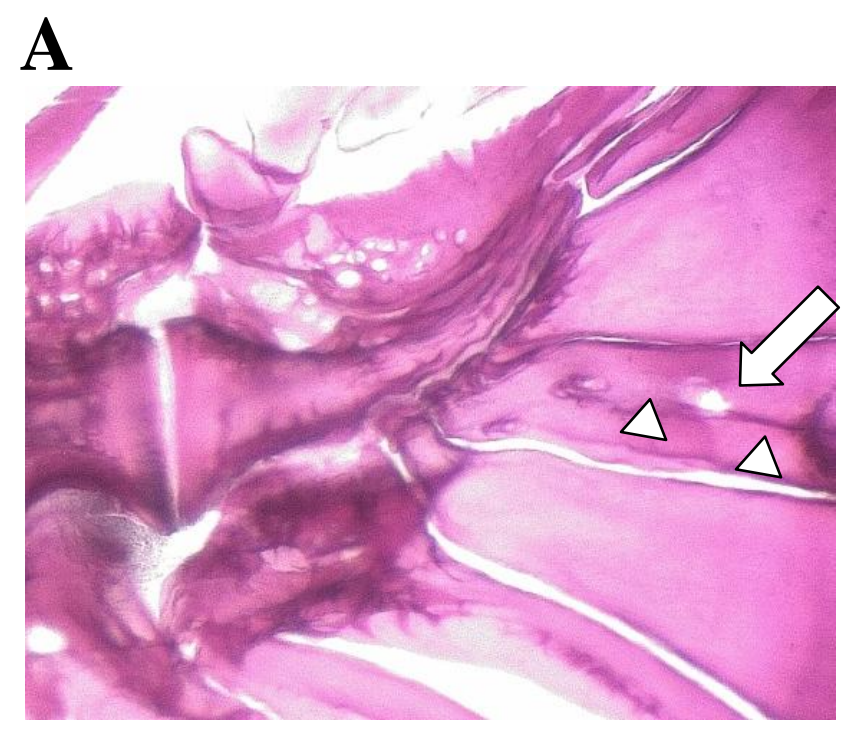

B

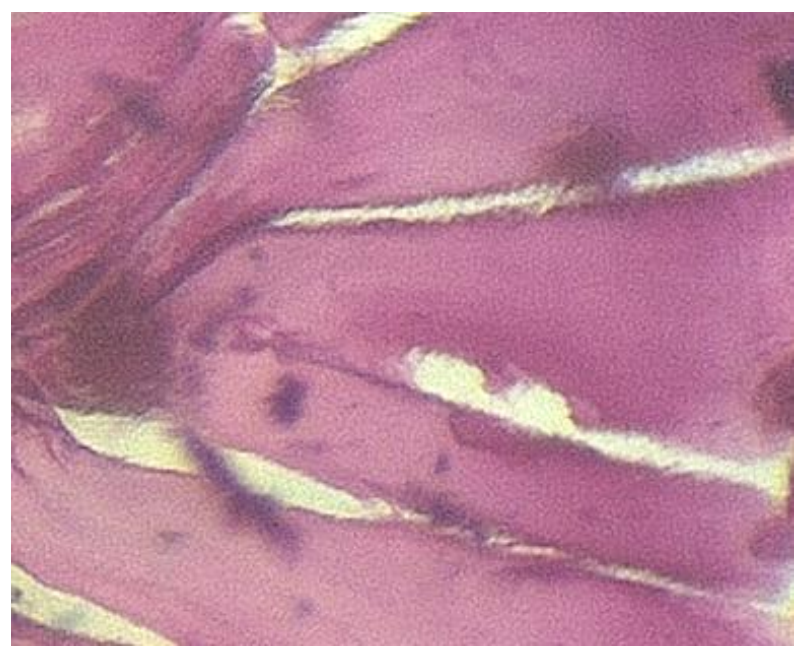

